\title{
Cerebral venous thrombosis in ulcerative colitis
}

\author{
Bindu Menon, Rajeev Goyal, Lalit Nihal' ${ }^{1}$ Rajasekhar Reddy² \\ Departments of Neurology, ${ }^{1}$ Gastroenterology, ${ }^{2}$ Pathology, Narayana Medical College and Superspeciality Hospital, Nellore, Andhra Pradesh, India
}

\section{ABSTRACT}

Ulcerative colitis has been reported to show hyper coagulation leading to peripheral and rarely central thrombosis. A 35-year-old female was admitted with chief complaints of increased frequency of bloody diarrhea, abdominal pain, and weight loss for 2 months. The patient was diagnosed to have ulcerative colitis after sigmoidoscopy and biopsy and she was started on treatment. Two days later, the patient developed headache and seizures. Magnetic resonance imaging of brain showed cerebral venous thrombosis with venous infarcts. A high index of clinical suspicion is needed to diagnose this uncommon condition so that appropriate treatment can be initiated.

Key words: Cerebral venous thrombosis, irritable bowel syndrome, ulcerative colitis

\section{Introduction}

Cerebral venous thrombosis (CVT) presents with protean manifestations which may mimic several other neurological disorders and hence is occasionally misdiagnosed. Ulcerative colitis is a type of chronic inflammatory bowel disease (IBD) that affects the lining of the large intestine (colon) and rectum. The etiology is unknown. Patients with active IBD are at an increased risk of thromboembolism which includes deep vein thrombosis, pulmonary embolism, cerebral venous thrombosis, and rarely arterial thrombosis. ${ }^{[1]}$ We report a patient who presented with gastrointestinal symptoms and during the acute phase of the illness developed cerebral venous thrombosis.

A 35-year-old female was admitted with chief complaints of increased frequency of bowels and abdominal pain for 2 months. The patient had a history of significant weight loss. The patient used to have frequency of 7-8 motions with blood and mucus. She had no history of fever, night sweats, and arthritis. The patient was not on any medication nor had any other major ailment in the past. On physical examination, the patient was moderately

\begin{tabular}{|l|l|}
\hline \multicolumn{2}{|c|}{ Access this article online } \\
\hline Quick Response Code: & Website: \\
\hline & www.ruralneuropractice.com \\
\cline { 2 - 3 } & \\
\hline & \\
\hline
\end{tabular}

built and pale. She was afebrile; pulse 90/minute and blood pressure was $110 / 60 \mathrm{~mm} \mathrm{Hg}$. There was no lymphadenopathy. The chest was clear. Her abdomen was flat, with normal bowel sounds. There was mild tenderness diffusely but no rebound tenderness or guarding. Rectal examination showed perianal ulcers. Sigmoidocopy revealed perianal fistula and perianal ulcers suggestive of inflammatory bowel disease. Biopsies were taken from both the affected and normal-appearing areas. H and E stain of a colonic biopsy [Figure 1a showed a crypt abscess (arrow) and goblet cell loss (arrow head) confirming ulcerative colitis. The patient was started on steroids, 5-aminosalycillic acid, and sulfasalazine. Three days later she developed headache. Headache was insidious, progressive, holocranial that gradually became severe in the next 2 days. She developed two episodes of generalized seizures and became drowsy. Fundus showed early papilledema. She had no cranial or limb weakness but had extensor plantar.

Laboratory findings showed hemoglobin $8.4 \mathrm{~g} \%$; the white blood cell count was slightly elevated at 11,200/ $\mathrm{mm}^{3}$. Erythrocyte sedimentation rate was $25 \mathrm{~mm}$ in the first hour, prothrombine time (PT) of 13.4 seconds (reference range: 12.0 seconds), with an INR at 1.5, and a normal PTT at 38.4 seconds (reference range: 32-40 seconds). Liver function, renal function, serum electrolytes were within normal limits.

Noncontrast-computed tomography scan of the head was normal. With suspicion of venous thrombosis,

\section{Address for correspondence:}

Dr. Bindu Menon, Department of Neurology, Narayana Medical College and Superspeciality Hospital, Chintareddypalem, Nellore, Andra Pradesh, India. E-mail: bneuro_5@rediffmail.com 
magnetic resonance venogram of the brain was done. Venogram showed loss of flow signal in superior sagittal sinus [Figure 1b], left transverse sinus, and sigmoid sinus [Figure 1c]. MRI brain showed left frontal infarct [Figure 1d]. The patient was started on low molecular heparin $0.4 \mathrm{ml}$ subcutaneous 12 hourly, phenytoin, intravenous dexamethasone, and antiedema measures. Her symptoms subsided and she developed no further complications. She was discharged on aspirin, sulfasalazine, steroids, and phenytoin. Her prothrombotic workup such as factor V Leiden, protein C and S, factor VIII, and antithrombin III was normal after 6 months. Antinuclear antibodies and anticardiolipin antibodies were negative and homocysteine levels were normal. She is asymptomatic and on regular follow-up.

\section{Discussion}

Cerebral venous thrombosis (CVT) is a rare but potentially devastating complication of IBD. The diagnosis needs to be considered in any patient with IBD presenting with neurological symptoms. Ulcerative colitis is a type of chronic inflammatory bowel disease (IBD) that affects the lining of the large intestine (colon) and rectum. The etiology is unknown. Ulcerative colitis may affect any age group, although there are peaks at ages $15-30$ and then again at ages $50-70$. A total of $40 \%$
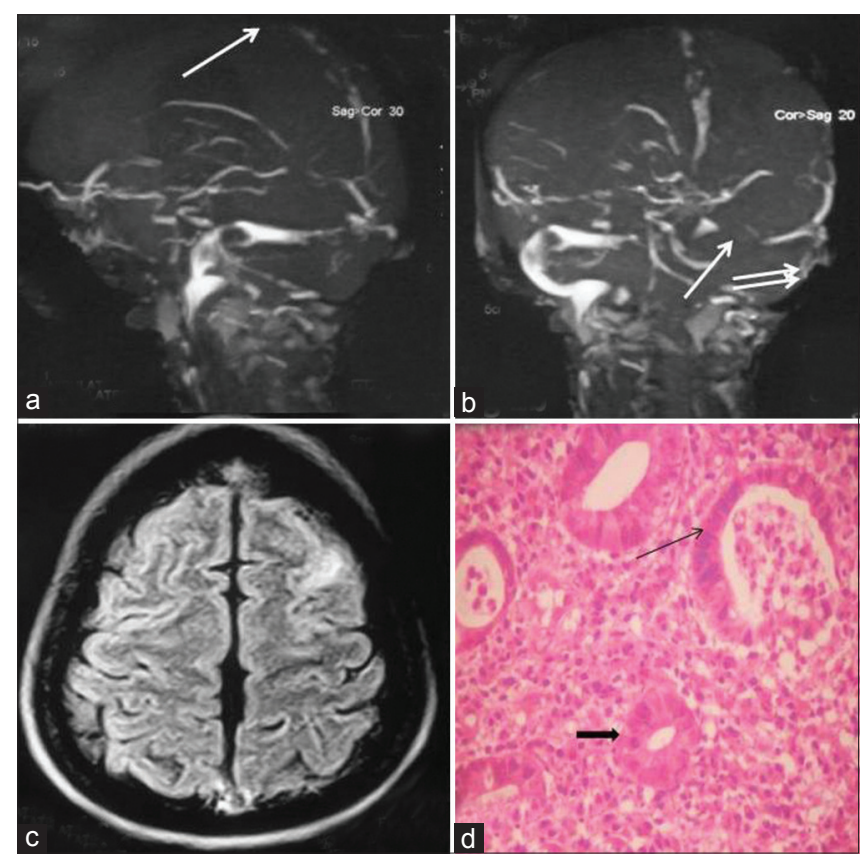

Figure 1: (a) MR venogram of the brain showing loss of flow signal in superior sagittal sinus (b) MR venogram of the brain showing loss of flow signal in left transverse sinus and sigmoid sinus (c) MRI axial sections of the brain shows hyperintense signal in the left frontal region suggestive of infarct (d) $\mathrm{H}$ and $\mathrm{E}$ stain of a colonic biopsy showing a crypt abscess (arrow) and goblet cell loss (arrow head) confirming ulcerative colitis of adult patients have extra-intestinal manifestations in skin, joints, bones, lungs, blood, eyes, kidneys, liver, and peripheral, and central nervous system. ${ }^{[2]}$ Incidence of thrombosis is $6.5 \%$ in patients with active IBD. ${ }^{[3]} \mathrm{CVT}$ is more common in UC than in Crohn's disease. ${ }^{[4]}$

Various mechanisms have been postulated for thrombosis in UC which include hypercoagulation (elevated FVIII, fibrinogen, decrease in antithrombin, protein S and protein C), hypofibrinolysis [elevated PAI-1 and lipoprotein (a)], platelet abnormalities, endothelial dysfunction (increased von Willebrand factor), and immunological abnormalities (antiphosphlipid antibodies).$^{[5]}$

Our patient had a negative prothrombotic workup on follow-up 6 months later. Probably in the acute phase of the illness patient may have had a hypercoagulable state. Cerebral thrombosis is a matter of high concern as it can lead to high mortality. Hence a high index of suspicion should be there in the case of headache which is increasing in severity as was present in this case. Clinical manifestations of CVT can vary from headache to major neurological manifestations. The usual presenting complaint is headache in $70-90 \%$ of cases. ${ }^{[6]}$ Patients may present with idiopathic intracranial hypertension with headache, papilledema and visual disturbances, focal neurological deficit, and headache or seizures. Seizures are a common manifestation occurring in about $40 \%$ of cases. ${ }^{[7]}$ Focal neurological deficits are also present but were not seen in our patient though her imaging showed venous infarct. Treatment of the condition is by antiedema measures and heparin. ${ }^{[8]}$ Steroids reduce the intracerebral edema and are also indicated in the active cases of ulcerative colitis. Anticoagulant in the form of low molecular weight heparin is indicated in CVT, even in cases with intracerebral bleed to dissolve the thrombus and decreases the thrombus spread..$^{[9]}$ Our patient improved on treatment and is asymptomatic since last 2 years on follow-up. CVT can be fatal in IBD if not promptly diagnosed. This case report signifies the importance of considering the diagnosis of CVT in a case of IBD.

\section{References}

1. Talbot RW, Heppell J, Dozois RR, Beart RW Jr. Vascular complications of inflammatory bowel disease. Mayo Clin Proc 1986;61:140-5.

2. Ozdil S, Akyuz F, Pinarbasi B, Demir K, Karaca C, Boztas G, et al. Ulcerative colitis: Analyses of 116 cases (do extraintestinal manifestations effect the time to catch remission?). Hepatogastroenterology 2004;51:768-70.

3. Twig G, Zandman-Goddard G, Szyper-Kravitz M, Shoenfeld Y. Systemic thromboembolism in inflammatory bowel disease: Mechanisms and clinical applications. Ann N Y Acad Sci 2005;1051:166-73.

4. Umit H, Asil T, Celik Y, Tezel A, Dokmeci G, Tuncbilek N, et al. Cerebral 
sinus thrombosis in patients with inflammatory bowel disease: A case report. World J Gastroenterol 2005;11:5404-7.

5. Danese S, Papa A, Saibeni S, Repici A, Malesci A, Vecchi M. Inflammation and coagulation in inflammatory bowel disease: The clot thickens. Am J Gastroenterol 2007;102:174-86.

6. Ameri A, Bousser MG. Cerebral venous thrombosis. Neurol Clin 1992;10:87-111.

7. Ferro JM, Canhão P, Bousser MG, Stam J, Barinagarrementeria F; ISCVT Investigators. Early seizures in cerebral vein and dural sinus thrombosis: Risk factors and role of antiepileptics. Stroke 2008;39:1152-8.
8. Guenther G, Arauz A. Cerebral venous thrombosis: A diagnostic and treatment update. Neurologia 2011;26:488-98.

9. Coutinho JM, Ferro JM, Canhão P, Barinagarrementeria F, Bousser MG, Stam J; ISCVT Investigators. Unfractionated or low-molecular weight heparin for the treatment of cerebral venous thrombosis. Stroke 2010;41:2575-80.

How to cite this article: Menon B, Goyal R, Nihal L, Reddy R. Cerebral venous thrombosis in ulcerative colitis. J Neurosci Rural Pract 2013;4:84-6.

Source of Support: Nil. Conflict of Interest: None declared. 